\title{
ACE: an efficient and sensitive tool to detect insecticide resistance-associated mutations in insect acetylcholinesterase from RNA-Seq data
}

Dianhao Guo ${ }^{1,2+}$, Jiapeng Luo ${ }^{1,3+}$, Yuenan Zhou ${ }^{1}$, Huamei Xiao ${ }^{4}$, Kang He ${ }^{1}$, Chuanlin Yin ${ }^{1}$, Jianhua Xu ${ }^{4}$ and Fei $\mathrm{Li}^{1^{*}}$

\begin{abstract}
Background: Insecticide resistance is a substantial problem in controlling agricultural and medical pests. Detecting target site mutations is crucial to manage insecticide resistance. Though PCR-based methods have been widely used in this field, they are time-consuming and inefficient, and typically have a high false positive rate. Acetylcholinesterases (Ace) is the neural target of the widely used organophosphate (OP) and carbamate insecticides. However, there is not any software available to detect insecticide resistance associated mutations in RNA-Seq data at present.

Results: A computational pipeline ACE was developed to detect resistance mutations of ace in insect RNA-Seq data. Known ace resistance mutations were collected and used as a reference. We constructed a Web server for ACE, and the standalone software in both Linux and Windows versions is available for download. ACE was used to analyse 971 RNA-Seq data from 136 studies in 7 insect pests. The mutation frequency of each RNA-Seq dataset was calculated. The results indicated that the resistance frequency was 30\%-44\% in an eastern Ugandan Anopheles population, thus suggesting this resistance-conferring mutation has reached high frequency in these mosquitoes in Uganda. Analyses of RNA-Seq data from the diamondback moth Plutella xylostella indicated that the G227A mutation was positively related with resistance levels to organophosphate or carbamate insecticides. The wasp Nasonia vitripennis had a low frequency of resistant reads $(<5 \%)$, but the agricultural pests Chilo suppressalis and Bemisia tabaci had a high resistance frequency. All ace reads in the $30 \mathrm{~B}$. tabaci RNA-Seq data were resistant reads, suggesting that insecticide resistance has spread to very high frequency in $B$. tabaci.
\end{abstract}

Conclusions: To the best of our knowledge, the ACE pipeline is the first tool to detect resistance mutations from RNASeq data, and it facilitates the full utilization of large-scale genetic data obtained by using next-generation sequencing.

Keywords: RNA-Seq data, Insecticide resistance, Mutations, Ace, Detection

\section{Background}

Insect pests are closely connected to human affairs, and they damage approximately one third of the agricultural, forestry and livestock production worldwide and consume tens of billions of dollars annually [1]. Although several alternative strategies such as transgenic crops and biological control measures have recently been

\footnotetext{
* Correspondence: lifei18@zju.edu.cn

${ }^{\dagger}$ Equal contributors

'Ministry of Agriculture Key Lab of Molecular Biology of Crop Pathogens and Insects, Institute of Insect Science, Zhejiang University, 866 Yuhangtang Road, Hangzhou 310058, China

Full list of author information is available at the end of the article
}

implemented in pest control, the use of chemical insecticides remains the most efficient and economic approach. However, use of insecticides has led to resistance, which is one of the best examples of rapid micro-evolution and has challenged the application of insecticides [2, 3]. The study of insecticide resistance is important because of its relevance to food safety, ecological safety and environmental pollution.

Target insensitivity is one of the main mechanisms conferring insecticide resistance. Because of long-term selection by insecticides, mutations are introduced into the active sites of genes that encode proteins that are the 
targets of insecticides. Given that the mutation frequency in the field population is a reliable indicator of the resistance level, monitoring resistance mutations in a field population of insect pests is highly important [4]. PCR-based methods such as PCR amplification of specific alleles (PASA) [5] and PCR-RFLP [6] are classical approaches that have been widely used. However, PCRbased methods have some disadvantages such as they are time-consuming and inefficient [7-10].

Acetylcholinesterases (ace, EC 3.1.1.7) are the target of OP and carbamate insecticides, which have been used to control nearly all notorious agricultural and medical pests such as rice stem borers, Colorado potato beetles, mosquitoes and houseflies. Two ace which encoding different ACHE proteins have been found in all insects except the Cyclorrhapha suborder of Diptera [11]. The mutation of ace to an insensitive form has been demonstrated as an important mechanism for insecticide resistance in many pests. In Drosophila melanogaster, 4 point mutations (F115S, I199V, G303A, and F368Y) have been identified to confer insecticide resistance [12]. Five mutations (V180 L, G262A, G262 V, F327Y, and G365A) in the ace of the housefly, Musca domestica, either singly or in combination, confer different levels of insecticide resistance [13]. The G119S mutation, which lies within the active "gorge" in ace-1 of Anopheles gambiae and Culex pipiens, results in resistance to propoxur [14]. Many resistance-associated mutations have also been identified in other insect pests [15-17].

RNA sequencing (RNA-Seq) provides the whole transcriptome of a biological sample at a given time by using a shotgun strategy with next-generation sequencing (NGS) techniques. The raw reads of the RNA-Seq data contain information on transcript abundance, alternative splicing and single nucleotide polymorphisms (SNP)/ mutations $[18,19]$. RNA-Seq data are useful in studying insecticide resistance, but unfortunately are not fully utilized at present. Most RNA-Seq data are used as a resource to obtain gene sequences. Here, to fully use RNA-Seq data to study insecticide resistance, we developed a pipeline, ACE, to detect resistance-associated mutations in ace genes from RNA-Seq data and applied this pipeline to estimate the mutation frequencies in 7 important insect pests.

\section{Results}

\section{Evolution analysis of two ace genes in insects}

By searching against the GenBank database and using BLASTP against the InsectBase database with 15 known ACHE protein sequences, we collected 62 ace 1 from 62 species and 70 ace 2 from 70 species. These ace genes were from 9 orders, including Siphonaptera, Diptera, Hymenoptera, Hemiptera, Coleoptera, Phthiraptera, Psocoptera, Blattodea and Lepidoptera (Additional file 1:
Table S1). To the best of our knowledge, this is the most comprehensive list of insect ace to date. Phylogenetic analysis using the neighbour-joining method indicated that most insects have two aces, except for the Cyclorrhapha suborder of Diptera (Fig. 1), suggesting that suggesting two ace were present before the diversification of insects. The loss of ace1 occurred in some Diptera insects.

\section{Insecticide resistance-associated mutations of ace}

We performed reference mining from 440 references to obtain a full list of insecticide resistance mutations of the ace in insects. Insect ACHEs were aligned with Torpedo californica ACHE (PDB ID code 1EA5), and the corresponding position of each mutation in T. californica ace was determined. In total, 14 mutations were found at 10 positions in ace1, and 22 mutations were found at 18 positions in ace2 (Fig. 2, Additional file 2: Table S2). Although there were several resistance mutations in both ace, most of the mutations occurred at 5 positions, 119, 201, 227, 290 and 331. These positions fall within the active gorge of $\mathrm{ACHE}$, thus demonstrating a common mechanism conferring insecticide resistance.

\section{ACE pipeline to detect resistance mutations of ace genes} We developed a pipeline, named ACE, to detect insecticide resistance mutations from RNA-Seq data (Fig. 3). First, the clean reads of the RNA-Seq data (Base-calling quality, Q30 $\geq 85 \%$ ) in standard Fastq format were mapped against the ace 1 or ace 2 of the species of interest by using Bowtie 2 with the default parameters [20]. This step identified all reads corresponding to ace. Second, we constructed a mutation site profile of ace for each insect, which consisted of susceptible and resistant fragments (11 nucleotides in length) covering each mutation site. We determined the cutoff of $11 \mathrm{bp}$ based on a pilot survey. If we use a long segment of $>13 \mathrm{bp}$, some reads will be lost. However, if we used a short segment $<9 \mathrm{bp}$, it will be mapped to other non-ace transcripts. Third, the reads that mapped to ace were used to scan for susceptible and resistant fragments with a customized Perl script. The reads containing susceptible fragments were treated as susceptible reads, and those containing resistant fragments were resistant reads. The percentages of susceptible or resistant reads were then calculated.

$$
\begin{aligned}
\text { Resistance frequency } & =\frac{\text { Count of resistant reads }}{\text { count of resistant reads }} \\
& + \text { count of susceptible reads }
\end{aligned}
$$

\section{Implementation}

We developed a standalone software and a Web server for the ACE pipeline. The standalone software is 


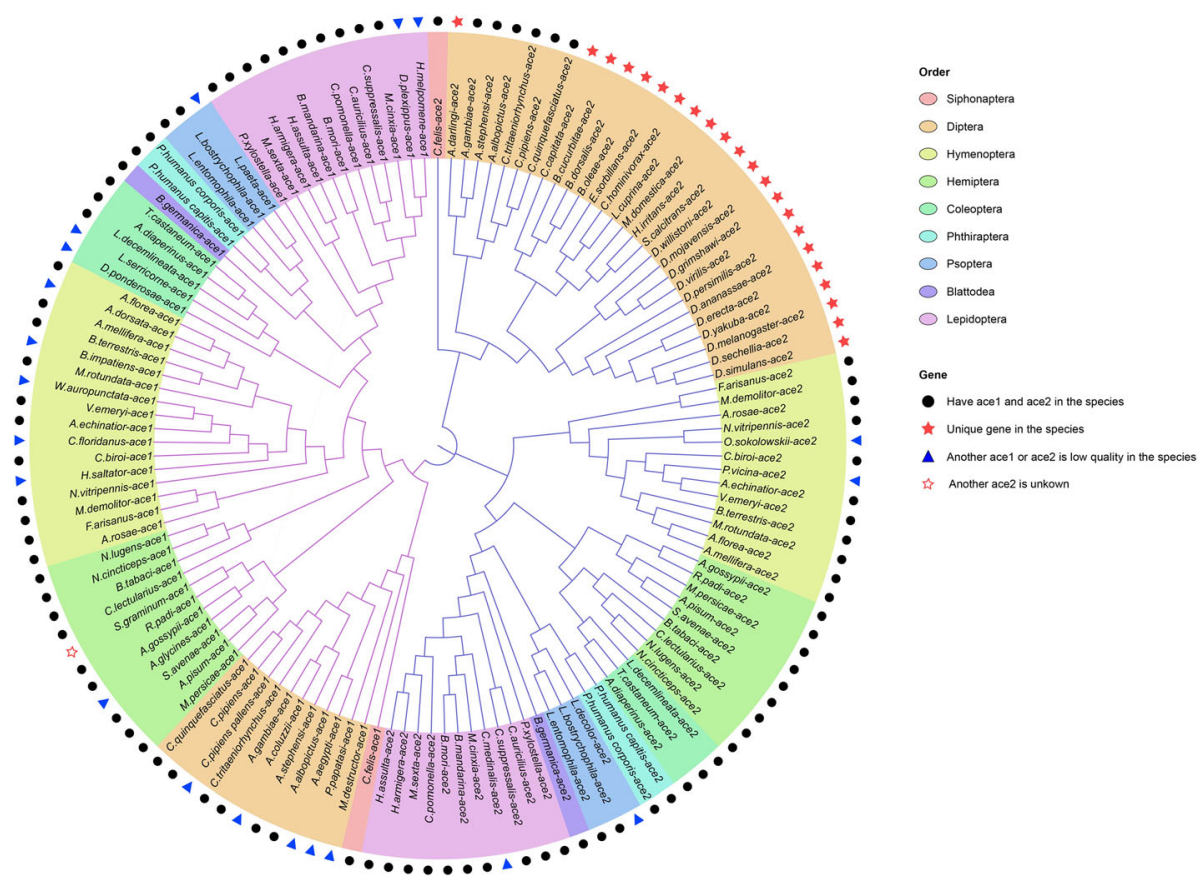

Fig. 1 Evolution analysis of two ace genes in insects. The amino acid sequences were used for the phylogenetic analysis. The sequence accession numbers are given in Table S1. The neighbour-joining method was used with 1000 replicates. The evolution tree indicated that most insects have two ace genes, except for the Cyclorrhapha suborder of Diptera

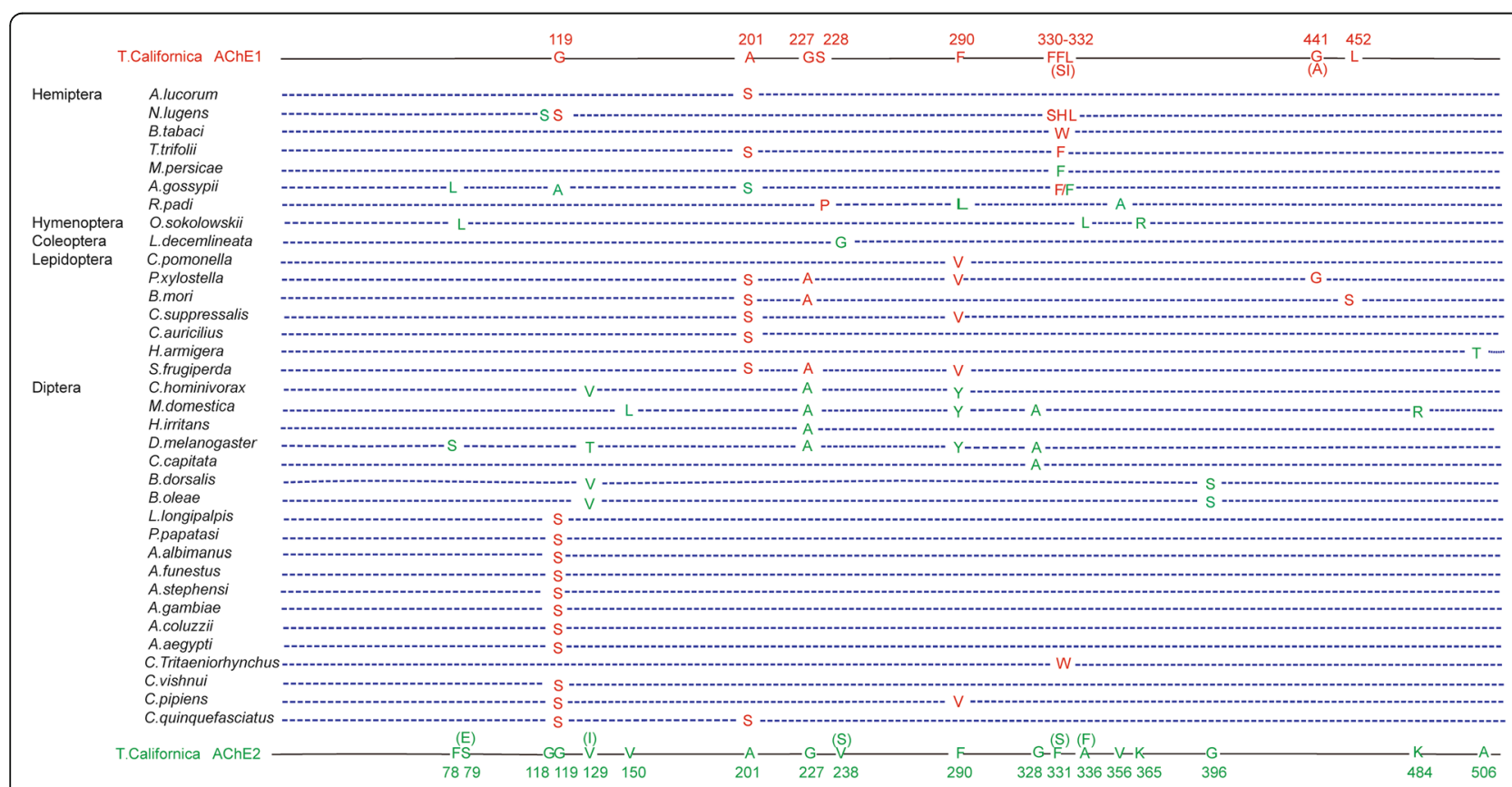

Fig. 2 Resistance mutation profile of insect acetylcholinesterases. The mutations were collected from 440 published references. Insect AChEs were aligned with Torpedo californica AChE (PDB ID code 1EA5) and the corresponding position of each mutation in Torpedo AChE was determined 


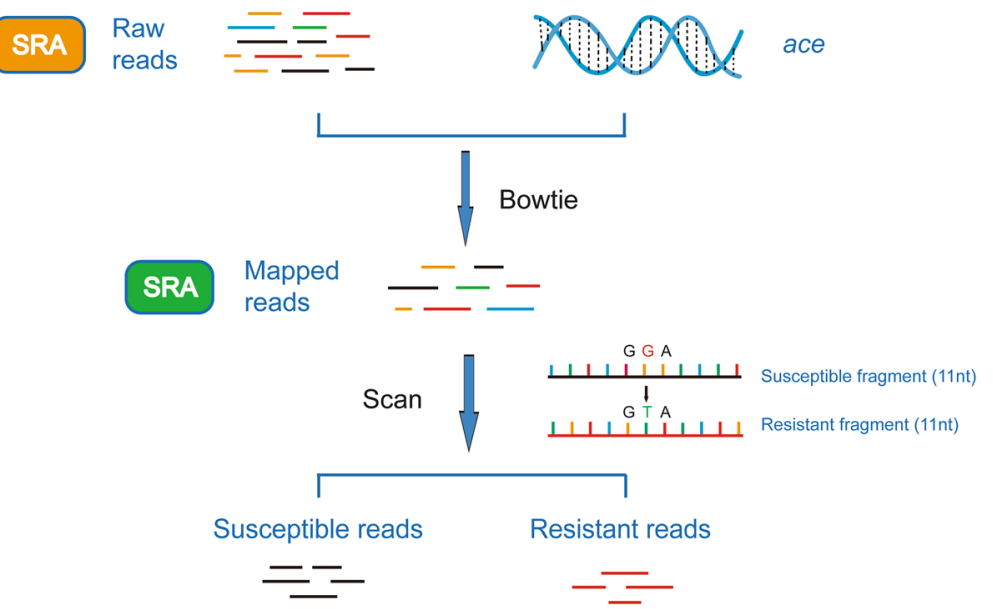

Fig. 3 The principles of the ACE pipeline. Raw reads were mapped with insect ace gene sequences by using Bowtie 2. Then, the resistant fragment $(11 \mathrm{nt})$ and susceptible fragment $(11 \mathrm{nt})$ flanking the mutation sites were used to scan the reads mapped with the insect ace gene. The scanned reads were divided into two types: resistant reads and susceptible reads

available for download. The Web server can be accessed at http://genome.zju.edu.cn/software/ace/. The Apache HTTP server was deployed in a Red Hat 6.5 Linux operating system. The Web pages were written by using HTML and Cascading Style Sheets (CSS). We also used Asynchronous JavaScript and XML (AJAX) to achieve some of the dynamic parts of the Web pages. The PHP script calls the ACE program, which runs online when the HTTP server receives the request from a Web client. The standalone version was built on the ultrafast short read mapping program Bowtie 2 [21]. All parameters were set as the default except using "-no-unal" as an additional parameter. Both Linux and Windows versions of the ACE standalone software are available. ACE is rapid and took only 5 min to process the 5 Gb RNA-Seq data on a Red Hat server (Dell X3250, Red Hat 6.5 Linux 64 bits, $3.1 \mathrm{GHz} 4 \mathrm{CPU}$ each with 4 cores, $32 \mathrm{G}$ memory).

\section{Application of ACE to analysis of RNA-Seq data in 7 insect pests}

We used the ACE pipeline to analyse the RNA-Seq data of 7 insect pests, including An. gambiae, C. floridanus, $N$. vitripennis, C. suppressalis, $P$. xylostella, $N$. lugens and B. tabaci (Additional file 3: Table S3). In An. gambiae, the major vector of Plasmodium falciparum malaria, we obtained RNA-Seq data from 468 samples, of which 20 were from an eastern Ugandan population. Since the G119S mutation of ace1 has been reported to confer insecticide resistance, we identified resistant reads from all 468 RNA-Seq data of An. gambiae by using the ACE pipeline. The results indicated that the resistance frequency was $30 \%-44 \%$ in the eastern Ugandan population, suggesting that the resistance in the Ugandan Anopheles population has reached very high frequency
(Fig. 4). There were no significant differences between male and female An. gambiae (t-test, $P$-values $=0.566$, Fig. 5). Surprisingly, we found significant differences among different developmental stages of the Pimperena strain of An. gambiae. The resistance frequency was significantly higher in late larvae and pupae than in the embryo and adult stages (One-way ANOVA test, $\mathrm{F}=27.621, p$-value $=8.186 \mathrm{E}-7$, Fig. 6$)$. The high resistance frequency in the late larvae and pupae stages enables mosquitoes to survive the insecticide treatment. However, mutations often incur high fitness costs such as low fecundity. Our results showed that the mosquito population had a low resistance frequency at the adult

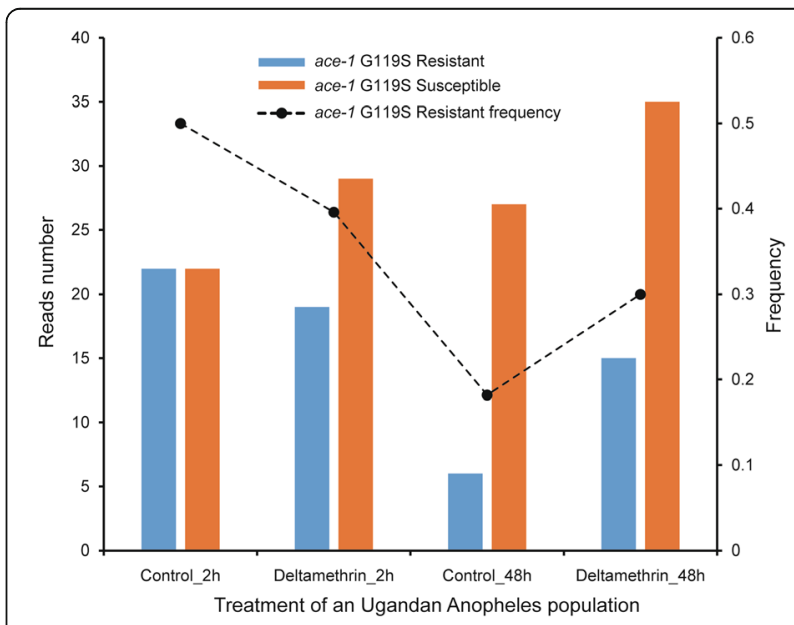

Fig. 4 The resistance frequency of four samples of a Ugandan population of Anopheles gambiae. The control was an untreated population which has high resistance to pyrethroids. The other two groups were treated with deltamethrin at $2 \mathrm{~h}$ or $48 \mathrm{~h}$ post treatment. The G119S mutation of acel was detected. The results indicated that the resistance level in this Ugandan Anopheles population was very high 


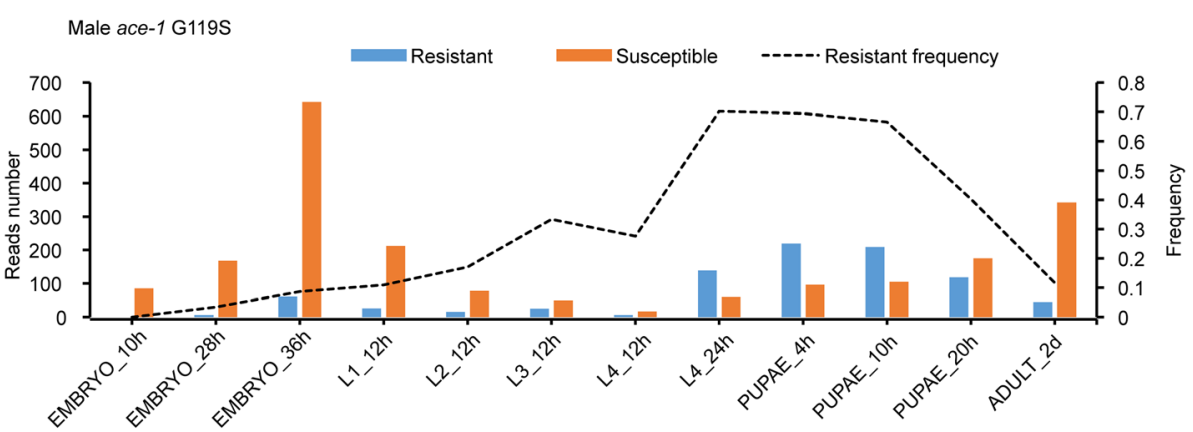

Developmental stages

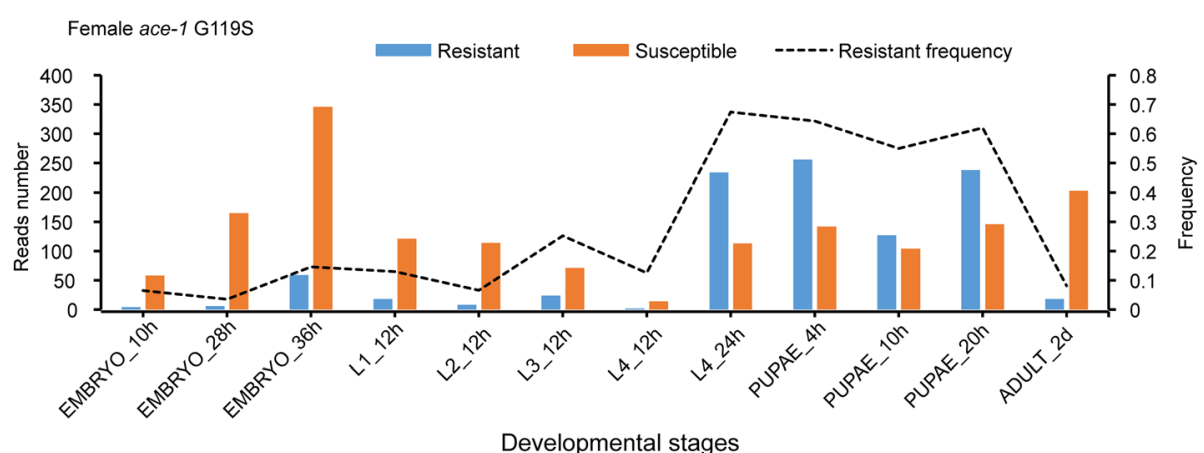

Fig. 5 Detection of the G119S mutation in the different sexes of A. gambiae. There were no significant differences in the resistance frequency between males and females (t-test, $P=0.566$ ). The sequencing depths were different in various samples, the read counts were varied. We recommend using the mutation frequency

stage, thus enabling the mosquitoes to produce offspring with a relatively high fitness. The detail mechanism is worthy of further investigation.

Analyses of the P. xylostella RNA-Seq data indicated that the G227A mutation was positively related with resistance levels to organophosphate or carbamate insecticides (F-test, $p<0.0 .5$ ), whereas the A201S mutation was only a minor contributor (F-test, $p>0.0 .5$, Fig. 7). The wasp $N$. vitripennis and ant Camponotus floridanus had a low frequency of resistant reads $(<5 \%$, Table 1$)$. However, the agricultural pests $C$. suppressalis and $B$. tabaci had a high resistance frequency. Approximately
$70 \%$ of C. suppressalis ace reads were resistant (Table 1), and most of the B. tabaci RNA-Seq data had $>90 \%$ resistant ace reads. All ace reads in the $30 \mathrm{~B}$. tabaci RNASeq data were resistant reads, suggesting that $B$. tabaci has developed extremely high resistance to insecticides (Additional file 4: Table S4).

\section{Discussion}

Insecticide resistance is a major problem in agriculture. Target insensitivity induced by mutations has been well studied. In past decades, several target site mutations have been identified in the insect ace gene. PCR-based

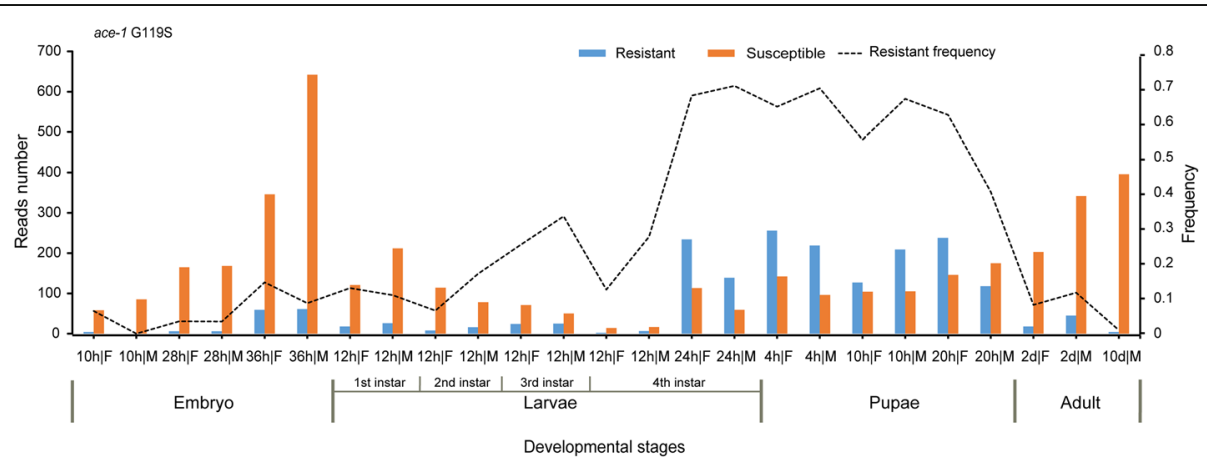

Fig. 6 Detection of the G119S mutation in the different developmental stages of A. gambiae. The late 4th instar larvae and pupae stages had higher resistance frequencies than the embryo and adult stages (One-way ANOVA test, $p<0.01$ ) 


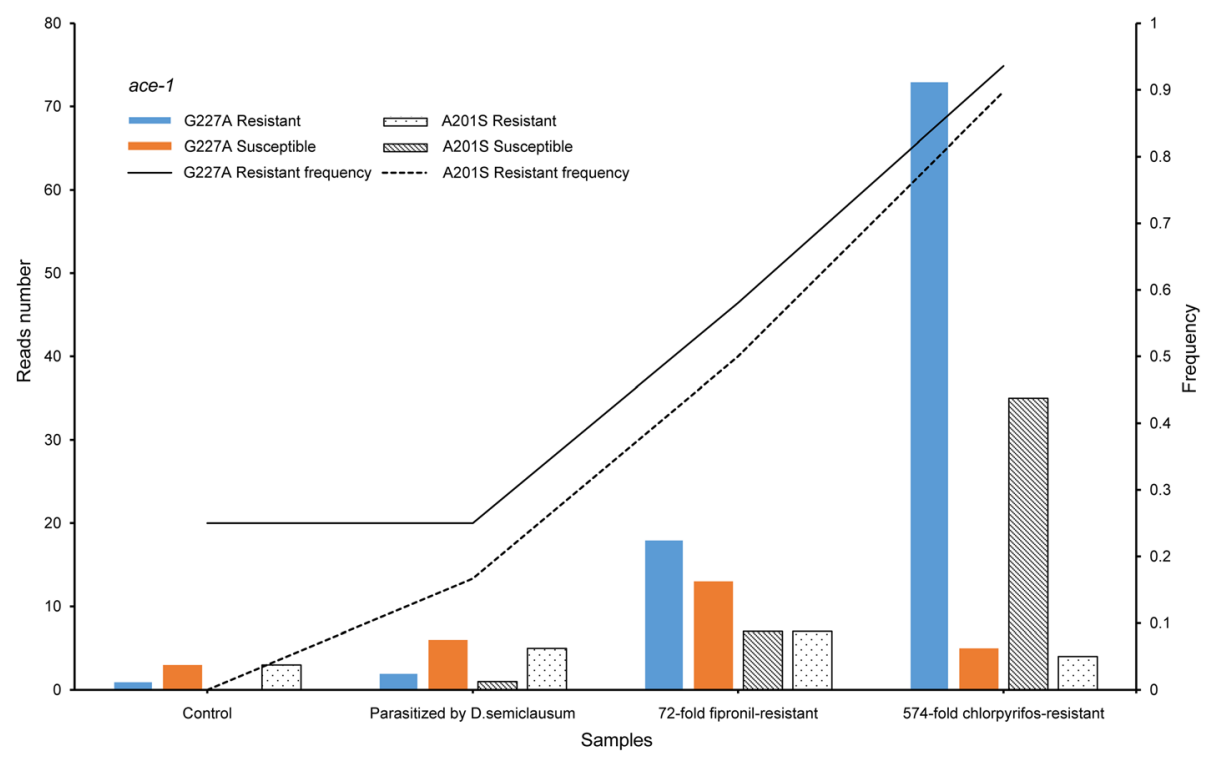

Fig. 7 The frequencies of the G227A and A201S mutations in the different samples of Plutella xylostella. The G227A mutation was positively associated with resistance to OP or carbamate insecticides, whereas the A201S mutation was not a major contributor

methods have been developed to detect resistance mutations [3, 4, 21]. Recently, RNA-Seq data obtained by using NGS techniques provide a valuable means to study insecticide resistance. Millions of raw reads can be obtained in a single run, thus enabling detection of low frequency mutations. Here, we developed a pipeline, ACE, to identify resistance-associated mutations by using RNA-Seq data. ACE has a high sensitivity and can detect resistant reads at low frequency. It should be noted that very low frequencies of resistant reads should be interpreted with caution due to the possibility of genotyping errors. Owing to the rapid development of NGS techniques, the cost of RNA-Seq has significantly decreased. This pipeline is useful for monitoring resistanceassociated mutation(s) in field population by using RNA-Seq data. ACE is also applicable for detecting resistance mutations from the genome re-sequencing data.

The ACE pipeline was used to analyse RNA-Seq data from 7 insect pests. The results proved that the ACE pipeline can successfully detect resistance mutations from millions of reads. Calculating the resistance frequency from the RNA-Seq data of these insect pests

Table 1 The resistance frequencies of predicted from RNA-Seq data by ACE

\begin{tabular}{|c|c|c|c|c|c|c|c|c|c|c|}
\hline \multirow[t]{2}{*}{ Species } & \multirow{2}{*}{$\begin{array}{l}\text { SRA accession } \\
\text { number }\end{array}$} & \multicolumn{8}{|c|}{ Resistance frequency (\%) } & \multirow[t]{2}{*}{ References } \\
\hline & & $\begin{array}{l}\text { G118S } \\
\text { ace2 }\end{array}$ & $\begin{array}{l}\text { A201S } \\
\text { acel }\end{array}$ & $\begin{array}{l}\text { A201S } \\
\text { acel }\end{array}$ & $\begin{array}{l}\text { G227A } \\
\text { acel }\end{array}$ & $\begin{array}{l}\text { F290 V } \\
\text { acel }\end{array}$ & $\begin{array}{l}\text { F330S } \\
\text { acel }\end{array}$ & $\begin{array}{l}\mathrm{F} 331 \mathrm{H} \\
\text { acel }\end{array}$ & $\begin{array}{l}\text { S332 L } \\
\text { acel }\end{array}$ & \\
\hline \multirow[t]{7}{*}{ Nasonia vitripennis } & SRR1262367 & & & & & 1.9 & 1.4 & & & (Hoedjes, et al., 2015) [43] \\
\hline & SRR1262372 & & 3.6 & & & 2.1 & & & & \\
\hline & SRR1262376 & & & & 3.7 & & & & & \\
\hline & SRR1262379 & & & & & & 2.4 & & & \\
\hline & SRR940321 & & & & & 1 & & 1.2 & & (Os, et al., 2013) [44] \\
\hline & SRR940323 & 66.7 & & & 2.7 & 0.9 & & & & \\
\hline & SRR1566027 & & & & & & & 4.8 & & (Wang, et al., 2015) [45] \\
\hline \multirow[t]{3}{*}{ Camponotus floridanus } & SRR1609918 & & & & 2.5 & & & & & (Gupta, et al., 2015) [46] \\
\hline & SRR330970 & & & & & 3.8 & & & & (Bonasio, et al., 2012) [47] \\
\hline & SRR490202 & & & & 0.9 & & & & & (Simola, et al., 2013) [48] \\
\hline \multirow[t]{3}{*}{ Chilo suppresssalis } & SRR651040 & & & 73.5 & & & & & & (Wu, et al., 2013) [49] \\
\hline & SRR2015503 & & & 70.8 & & & & & & (Xu, et al., 2015) [50] \\
\hline & SRR1200447 & & & & & & & & & (Cao, et al., 2014) [51] \\
\hline
\end{tabular}


confirmed the importance of target site mutations in conferring insecticide resistance. Large-scale level analyses also provided new insights into the evolution of and changes in resistance mutations. We found that the resistance mutation frequency changed during insect development. This change has not been previously reported and is worthy of further investigation.

As a tool to detect resistance-associated mutations from RNA-Seq data, we plan to develop additional integrated applications for ACE to address the following areas. First, development of insecticide resistance is a complex system. Different insecticides have various targets: organophosphate and carbamate insecticides target AChE; pyrethroids insecticides target sodium channels; neonicotinoid insecticides target nicotinic acetylcholine receptors (nAChR); and diamide insecticides target ryanodine receptors (RyR). We wish to broaden the scope of ACE to detect resistance mutations in all target genes. Second, increased metabolism of insecticides, owing to overexpression of detoxification enzymes, is another important mechanism of insecticide resistance. We wish to develop ACE to examine the abundance of P450, GST and esterase genes, which have been reported to have important roles in conferring resistance [22, 23]. Third, cross-resistance provides important information to improve the prediction efficiency [24-27], which has been well studied in human $[28,29]$, we wish to integrate this information in the future. Last, it has been reported that multiple alterations of gene sequences, such as alternative splicing and RNA editing, are also involved in insecticide resistance. We plan to develop ACE to detect novel SNPs and other types of sequence changes.

\section{Conclusions}

A computational tool was developed to detect insecticide resistance-associated mutation of AChE from insect RNA-Seq data. Both the standalone software and the Web server of ACE were provided. Analyses of 971 RNA-Seq data from 136 studies in 7 insect pests provided new insights into insecticide resistance, suggesting that insecticide resistance mutation might be associate with development stage of insects. Large-scale detection of insecticide resistance mutation using ACE demonstrated that the insecticide resistance of the eastern Ugandan mosquito population and whitefly $B$. tabaci has reached extremely high level.

\section{Methods}

\section{Data sources}

The ace sequences were retrieved from GenBank of the National Centre for Biotechnology Information (NCBI) [30]. We selected the ace genes of 8 insects as the sequence references. These ace were confirmed by using PCR and gene function analysis in the published reports of other groups, including ace 2 in D. melanogaster (NP_476953), ace1 and ace 2 in Culex tritaeniorhynchus (BAD06210, BAD06209), ace1 and ace2 in Plutella xylostella (AAY34743, AAL33820), ace1 and ace2 in Chilo suppressalis (ABO38111, ABR24230), ace1 and ace 2 in Tribolium castaneum (ADU33189, ADU33190), ace1 and ace 2 in Rhopalosiphum padi (AAT76530, AAU11285), ace 1 and ace 2 in Aphis gossypii (AAM94376, AAM94375), and ace1 and ace 2 in Liposcelis bostrychophila (ACN78619, ABO31937). The amino acid sequences of these $15 \mathrm{ACHE}$ were used as the query sequences in BLASTP against the official gene set (OGS) in InsectBase $($ E-value $=1 \mathrm{e}-30)$. The best BLASTP hit was treated as the candidate ace. To ensure reliability, sequences less than 1800 bp were removed. All identified ACHEs were confirmed to have two conserved motifs (WIY(F)GGG and FGESAE). These steps yielded 62 ace 1 from 62 species and 70 ace 2 from 70 species (Additional file 1: Table S1).

A total of 971 RNA-Seq data from 136 studies in 7 insect pests (An. gambiae, C. floridanus, $N$. vitripennis, $C$. suppressalis, $P$. xylostella, $N$. lugens and $B$. tabaci) were downloaded from the Sequence Read Archive database (SRA) of NCBI [31]. The SRA accession numbers are given in Additional file 2: Table S2.

\section{Phylogenetic analysis}

The amino acid sequences of $\mathrm{AChE}$ were aligned using MUSCLE [32]. The phylogenetic relationships were inferred using the neighbour-joining method [33] with 1000 replicates. The bootstrap values are shown next to the branches [34]. The evolutionary distances were computed using the Kimura 2-parameter method [35] and expressed as the number of base substitutions per site. The analysis involved 132 nucleotide sequences. All positions containing gaps and missing data were eliminated. There were 1239 positions in the final dataset. A phylogenetic tree was constructed by MEGA 7 [36]. A consensus tree was displayed and edited with iTOL [37]. The tree was drawn to scale, with branch lengths in the same units as those of the evolutionary distances used to infer the phylogenetic tree.

\section{Collecting known ace resistance-associated mutations}

To collect the known ace resistance-associated mutations, we downloaded the references from NCBI PubMed by searching with the keywords ("insecticide resistance" [Abstract] AND acetylcholinesterase [Abstract]), yielding 440 references. Among these references, only 5 used transcriptome methods to determine ace sequences [38-42], and only one reference used raw reads to call SNPs by using SOAPsnp [39]. We manually extracted ace mutations conferring insecticide resistance, which yielded 14 mutations at 10 positions in ace 1 and 22 mutations at 18 positions in ace 2 . 


\section{Additional files}

Additional file 1: Table S1. The NCBI accession numbers of insect ace-1 and ace-2 genes

Additional file 2: Table S2. Resistance mutations in ace-1 and ace-2 of insects

Additional file 3: Table S3. The SRA accession numbers of 971 RNA-Seq data used for detecting mutations

Additional file 4: Table S4. The resistance frequency of mutation S331 W in different RNA-Seq data of Bemisia tabaci

\section{Abbreviations}

AChE: Acetylcholinesterases; AJAX: Asynchronous JavaScript and XML; CSS: Cascading Style Sheets; nAChR: nicotinic acetylcholine receptors; NCBI: National Center for Biotechnology Information; NGS: Next-generation sequencing; OGS: Official gene set; OP: Organophosphate; PASA: PCR amplification of specific alleles; RNA-Seq: RNA sequencing; RyR: Ryanodine receptors; SNP: Single nucleotide polymorphisms; SRA: Sequence Read Archive database

\section{Acknowledgments}

The authors wish to thank Jinmeng Guo and Wanyi Ye in Zhejiang University for kind assistance.

\section{Funding}

This work was funded by the National Basic Research Program of China [2013CB127600], the National Key Research and Development Program [2016YFC1200600, SQ2017ZY060102], and the Science and Technology Research Project of the Ministry of Education [V201308]

\section{Availability of data and materials}

The ace gene sequences used in this study are available in the NCBI GenBank. The official gene sets (OGS) of insects are available in the InsectBase. All RNASeq data are available in the NCBI SRA database.

Project name: ACE.

Project home page: http://genome.zju.edu.cn/software/ace/

Operating system: Platform-independent: Programming language:Perl. Other requirements: Perl (version 5.14 or later).

License: GPL.

\section{Authors' contributions}

FL conceived and designed the study. DHG conducted the study. JPL and CLY constructed the webserver, JPL and DHG completed the standalone software. YNZ joined in the evolutional analyses. $\mathrm{KH}$ improved the figures. $\mathrm{FL}$ and DHG wrote the manuscript. HMX completed the second-round revision of the manuscript. All authors reviewed the manuscript. All authors read approved the final manuscript.

Ethics approval and consent to participate

Not applicable.

\section{Consent for publication}

Not applicable.

\section{Competing interests}

The authors declare that they have no competing interests.

\section{Publisher's Note}

Springer Nature remains neutral with regard to jurisdictional claims in published maps and institutional affiliations.

\section{Author details}

'Ministry of Agriculture Key Lab of Molecular Biology of Crop Pathogens and Insects, Institute of Insect Science, Zhejiang University, 866 Yuhangtang Road, Hangzhou 310058, China. ${ }^{2}$ Department of Entomology, College of Plant Protection, Nanjing Agricultural University, Nanjing 210095, China. ${ }^{3}$ College of Computer Science and Technology, Nanjing Normal University, Nanjing
210023, China. ${ }^{4}$ College of Life Sciences and Resource Environment, Yichun University, Yichun 336000, China.

Received: 20 January 2017 Accepted: 22 June 2017

Published online: 10 July 2017

\section{References}

1. Oerke EC. Crop losses to pests. J Agric Sci. 2006;144:31-43.

2. Denholm I, Devine GJ, Williamson MS. Evolutionary genetics. Insecticide resistance on the move. Science. 2002;297(5590):2222-3.

3. Hemingway J, Field L, Vontas J. An overview of insecticide resistance. Science. 2002;298(5591):96-7.

4. Feng $X$, Yang $C$, Yang Y, Li J, Lin K, Li M, et al. Distribution and frequency of G119S mutation in ace-1 gene within Anopheles Sinensis populations from Guangxi, China. Malar J. 2015:14:470.

5. Yan HH, Xue CB, Li GY, Zhao XL, Che XZ, Wang LL. Flubendiamide resistance and bi-PASA detection of ryanodine receptor G4946E mutation in the diamondback moth (Plutella xylostella L.). Pestic Biochem Physiol. 2014; 115:73-7.

6. Singh OP, Bali P, Hemingway J, Subbarao SK, Dash AP, Adak T. PCR-based methods for the detection of L1014 kdr mutation in Anopheles culicifacies sensu lato. Malar J. 2009:8:154.

7. Gawad C, Koh W, Quake SR. Single-cell genome sequencing: current state of the science. Nat Rev Genet. 2016:17(3):175-88.

8. Van Keuren-Jensen K, Keats JJ, Craig DW. Bringing RNA-seq closer to the clinic. Nat Biotechnol. 2014;32(9):884-5.

9. Kim S, Misra A. SNP genotyping: technologies and biomedical applications. Annu Rev Biomed Eng. 2007:9:289-320.

10. Castle PE, Porras C, Quint WG, Rodriguez AC, Schiffman M, Gravitt PE, et al. Comparison of two PCR-based human papillomavirus genotyping methods. J Clin Microbiol. 2008:46(10):3437-45

11. Cha DJ, Lee SH. Evolutionary origin and status of two insect acetylcholinesterases and their structural conservation and differentiation. Evol Dev. 2015;17(1):109-19.

12. Mutero A, Pralavorio M, Bride JM, Fournier D. Resistance-associated point mutations in insecticide-insensitive acetylcholinesterase. Proc Natl Acad Sci U S A. 1994;91(13):5922-6.

13. Walsh SB, Dolden TA, Moores GD, Kristensen M, Lewis T, Devonshire AL, et al. Identification and characterization of mutations in housefly (Musca domestica) acetylcholinesterase involved in insecticide resistance. Biochem J. 2001;359(1):175-81.

14. Weill M, Fort P, Berthomieu A, Dubois MP, Pasteur N, Raymond M. A nove acetylcholinesterase gene in mosquitoes codes for the insecticide target and is non-homologous to the ace gene in drosophila. Proc Biol Sci. 2002 269(1504):2007-16.

15. Lee $\mathrm{SH}$, Kim YH, Kwon DH, Cha DJ, Kim JH. Mutation and duplication of arthropod acetylcholinesterase: implications for pesticide resistance and tolerance. Pestic Biochem Physiol. 2015;120:118-24.

16. Luo GH, Li XH, Zhang ZC, Liu BS, Huang SJ, Fang JC. Cloning of two Acetylcholinesterase genes and analysis of point mutations putatively associated with Triazophos resistance in Chilo auricilius (Lepidoptera: Pyralidae). J Econ Entomol. 2015;108(3):1289-97.

17. Wu S, Zuo K, Kang Z, Yang Y, Oakeshott JG, Wu Y. A point mutation in the acetylcholinesterase-1 gene is associated with chlorpyrifos resistance in the plant bug Apolygus lucorum. Insect Biochem Mol Biol. 2015;65:75-82.

18. Sacomoto GA, Kielbassa J, Chikhi R, Uricaru R, Antoniou P, Sagot MF, et al. KISSPLICE: de-novo calling alternative splicing events from RNA-seq data. BMC Bioinformatics. 2012;13(Suppl 6):S5

19. Yang $M$, Xu L, Liu Y, Yang P. RNA-Seq uncovers SNPs and alternative splicing events in Asian lotus (Nelumbo nucifera). PLoS One. 2015;10(4): e0125702.

20. Langdon WB. Performance of genetic programming optimised Bowtie2 on genome comparison and analytic testing (GCAT) benchmarks. BioData mining. 2015;8(1):1.

21. Langmead B, Salzberg SL. Fast gapped-read alignment with Bowtie 2. Nat Methods. 2012:9(4):357-9.

22. Rosenhauer M, Felsenstein FG, Piepho HP, Höfer M, Petersen J. Segregation of non-target-site resistance to herbicides in multiple-resistant Alopecurus myosuroides plants. Weed Res. 2015;55(3):298-308.

23. Wang B, Shahzad MF, Zhang Z, Sun H, Han P, Li F, et al. Genome-wide analysis reveals the expansion of Cytochrome P450 genes associated with 
xenobiotic metabolism in rice striped stem borer, Chilo Suppressalis. Biochem Biophys Res Commun. 2014;443(2):756-60.

24. Bel Y, Sheets JJ, Tan SY, Narva KE, Escriche B. Toxicity and binding studies of bacillus thuringiensis Cry1Ac, Cry1F, Cry1C and Cry2A proteins in the soybean pests Anticarsia gemmatalis and Chrysodeixis (Pseudoplusia) includens. Appl Environ Microbiol. 2017:83(11):e00326-17.

25. Wei Q, Mu XC, Wu SF, Wang LX, Gao CF. Cross-resistance to three phenylpyrazole insecticides and $A 2^{\prime} \mathrm{N}$ mutation detection of GABA receptor subunit in fipronil-resistant Laodelphax striatellus (Hemiptera: Delphacidae). Pest Manag Sci. 2017;73(8):1618-24.

26. Mu XC, Zhang W, Wang LX, Zhang S, Zhang K, Gao CF, et al. Resistance monitoring and cross-resistance patterns of three rice planthoppers, Nilaparvata lugens, Sogatella furcifera and Laodelphax striatellus to dinotefuran in China. Pestic Biochem Physiol. 2016;134:8-13.

27. Atencia MC, Perez MJ, Jaramillo MC, Caldera SM, Cochero S, Bejarano EE. First report of the F1534C mutation associated with cross-resistance to DDT and pyrethroids in Aedes aegypti from Colombia. Biomedica Rev del Inst Nac de Salud. 2016;36(3):432-7.

28. Riemenschneider $M$, Senge $R$, Neumann U, Hullermeier $E$, Heider D. Exploiting HIV-1 protease and reverse transcriptase cross-resistance information for improved drug resistance prediction by means of multilabel classification. BioData mining. 2016;9:10.

29. Heider D, Senge R, Cheng W, Hullermeier E. Multilabel classification for exploiting cross-resistance information in HIV-1 drug resistance prediction. Bioinformatics. 2013;29(16):1946-52.

30. Clark K, Karsch-Mizrachi I, Lipman DJ, Ostell J, Sayers EW. GenBank. Nucleic Acids Res. 2016:44(D1):D67-72.

31. Kodama Y, Shumway M, Leinonen R. International nucleotide sequence database $C$ : the sequence read Archive: explosive growth of sequencing data. Nucleic Acids Res. 2012;40(Database issue):D54-6.

32. Edgar RC. MUSCLE: multiple sequence alignment with high accuracy and high throughput. Nucleic Acids Res. 2004;32(5):1792-7.

33. Saitou N, Nei M. The neighbor-joining method: a new method for reconstructing phylogenetic trees. Mol Biol Evol. 1987;4(4):406-25.

34. Felsenstein J. Confidence limits on phylogenies: an approach using the bootstrap. Source: Evolution. 1985;39(4):783-91.

35. Kimura M. A simple method for estimating evolutionary rates of base substitutions through comparative studies of nucleotide sequences. J Mol Evol. 1980;16(2):111-20.

36. Kumar S, Stecher G, Tamura K. MEGA7: molecular evolutionary genetics analysis version 7.0 for bigger datasets. Mol Biol Evol. 2016;33(7):1870-4.

37. Letunic I, Bork P. Interactive tree of life (iTOL) v3: an online tool for the display and annotation of phylogenetic and other trees. Nucleic Acids Res. 2016;44(W1):W242-5.

38. Carvalho RA, Azeredo-Espin AM, Torres TT. Deep sequencing of new world screw-worm transcripts to discover genes involved in insecticide resistance. BMC Genomics. 2010;11:695.

39. He W, You M, Vasseur L, Yang G, Xie M, Cui K, et al. Developmental and insecticide-resistant insights from the de novo assembled transcriptome of the diamondback moth, Plutella xylostella. Genomics. 2012;99(3):169-77.

40. Silva AX, Jander G, Samaniego H, Ramsey JS, Figueroa CC. Insecticide resistance mechanisms in the green peach aphid Myzus persicae (Hemiptera: Aphididae) I: a transcriptomic survey. PLoS One. 2012;7(6):e36366.

41. Grigoraki L, Lagnel J, Kioulos I, Kampouraki A, Morou E, Labbe P, et al. Transcriptome profiling and genetic study reveal amplified Carboxylesterase genes implicated in Temephos resistance, in the Asian Tiger mosquito Aedes albopictus. PLoS Negl Trop Dis. 2015;9(5):e0003771.

42. Pan Y, Peng T, Gao X, Zhang L, Yang C, Xi J, et al. Transcriptomic comparison of thiamethoxam-resistance adaptation in resistant and susceptible strains of Aphis gossypii glover. Comp Biochem Physiol Part D Genomics Proteomics. 2015;13:10-5.

43. Hoedjes KM, Smid HM, Schijlen EG, Vet LE, van Vugt JJ. Learning-induced gene expression in the heads of two Nasonia species that differ in longterm memory formation. BMC Genomics. 2015;16:162.

44. Os A, Burgler S, Ribes AP, Funderud A, Wang D, Thompson KM, et al. Chronic lymphocytic leukemia cells are activated and proliferate in response to specific T helper cells. Cell Rep. 2013;4(3):566-77.

45. Wang X, Werren JH, Clark AG. Genetic and epigenetic architecture of sexbiased expression in the jewel wasps Nasonia vitripennis and giraulti. Proc Natl Acad Sci U S A. 2015;112(27):E3545-54.
46. Gupta SK, Kupper M, Ratzka C, Feldhaar H, Vilcinskas A, Gross R, et al. Scrutinizing the immune defence inventory of Camponotus floridanus applying total transcriptome sequencing. BMC Genomics. 2015;16:540.

47. Bonasio R, Li Q, Lian J, Mutti NS, Jin L, Zhao H, et al. Genome-wide and caste-specific DNA methylomes of the ants Camponotus floridanus and Harpegnathos saltator. Curr biol : CB. 2012;22(19):1755-64.

48. Simola DF, Ye C, Mutti NS, Dolezal K, Bonasio R, Liebig J, et al. A chromatin link to caste identity in the carpenter ant Camponotus floridanus. Genome Res. 2013;23(3):486-96

49. Wu SF, Sun FD, Qi YX, Yao Y, Fang Q, Huang J, et al. Parasitization by Cotesia chilonis influences gene expression in fatbody and hemocytes of Chilo Suppressalis. PLoS One. 2013;8(9):e74309.

50. Xu G, Wu SF, Wu YS, Gu GX, Fang Q, Ye GY. De novo assembly and characterization of central nervous system transcriptome reveals neurotransmitter signaling systems in the rice striped stem borer, Chilo Suppressalis. BMC Genomics. 2015;16:525.

51. Cao D, et al. Identification of candidate olfactory genes in Chilo Suppressalis by antennal transcriptome analysis. Int J Biol Sci. 2014;10(8):846-60.

\section{Submit your next manuscript to BioMed Central and we will help you at every step:}

- We accept pre-submission inquiries

- Our selector tool helps you to find the most relevant journal

- We provide round the clock customer support

- Convenient online submission

- Thorough peer review

- Inclusion in PubMed and all major indexing services

- Maximum visibility for your research

Submit your manuscript at www.biomedcentral.com/submit
) Biomed Central 